Sādhanā Vol. 40, Part 7, October 2015, pp. 2001-2019. (C) Indian Academy of Sciences

\title{
Electrodynamic energy harvester for electrical transformer's temperature monitoring system
}

\author{
FARID KHAN* and SHADMAN RAZZAQ \\ Institute of Mechatronics Engineering, University of Engineering \& Technology \\ Peshawar, Peshawar 25000, Pakistan \\ e-mail: dr_farid_khan@uetpeshawar.edu.pk
}

MS received 9 February 2015; revised 29 April 2015; accepted 6 June 2015

\begin{abstract}
The development of an electrodynamic energy harvester (EDEH) for operating a wireless temperature monitoring system for electrical transformer is reported in this work. Analytical modeling, fabrication and characterization of EDEH prototype are performed. The developed EDEH consists of a mild steel core, a wound copper coil and Teflon housing. COMSOL Multiphysics software is used to optimize the design of the harvester. The split-cylindrical design of the developed EDEH permitted the harvester to be wrapped around the output power cable of the electrical transformer without shutting-off the power or disconnecting the power cable. From the electrical transformer, at current levels of 27, 72 and $155 \mathrm{~A}$ in the main power line, the energy harvester produced maximum RMS load voltages of $0.356,1.09$ and $2.58 \mathrm{~V}$ respectively, when connected to $100 \Omega$ load resistance. However, at matching impedance of $24 \Omega$ (resistance of the coil), the EDEH produced the maximum power levels of 2.99, 19.66 and $112.03 \mathrm{~mW}$ for a cable currents of 27, 72 and $155 \mathrm{~A}$ respectively. The simulation results of the devised analytical model of the harvester are in good agreement with the experimental results. Moreover, at a cable current of 93 A, when the harvester is connected to the rectifying circuit, the optimum impedance shifted to $185 \Omega$ and the maximum power of $19 \mathrm{~mW}$ is generated at that load. The reduction in power generation is attributed to the power consumption of the rectifying circuit. When the rectified DC voltage is used to charge a $3.8 \mathrm{~V}$, Nickel-Cadmium (Ni-Cd) rechargeable battery, it took $3 \mathrm{~h}$ to completely charge the battery from 1 to $3.85 \mathrm{~V}$. With the charged battery a wireless temperature sensor node is successfully operated for monitoring the temperature of the electrical transformer.
\end{abstract}

Keywords. Electrical transformer; electrodynamic; energy harvester; self-powered system; temperature monitoring; wireless sensor node.

*For correspondence 


\section{Introduction}

Traditional electrical transformers often suffer failure because of overheating. In hot and humid countries, mostly during summer seasons when consumption of electricity increases with the usage of high power appliances in homes and in commercial areas, the risk of transformer's overheating enhances. The operating safe temperature of copper wounded electrical transformers is from 30 to $90^{\circ} \mathrm{C}$ Kennedy \& Gordner (2013). Overloading in transformers causes significant rise in temperature. If the overloading and overheating is not timely detected, it can lead to the catastrophic failure of a transformer and can cause heavy financial losses. With wireless temperature sensor nodes (WTSNs), the temperature of electrical transformer can be continuously monitored. Normally, batteries are utilized for the operation of WTSNs. However, due to the limited shelf life of batteries regular replacement and maintenance of WTSNs is required. Energy harvesting is a viable alternative to batteries in WTSNs. Energies, such as solar, acoustic, heat and vibration that is available in electrical transformer surroundings can be converted into electrical energy for WTSNs operation. In transformer, the changing magnetic field around the power line can also be utilized with an electrodynamic energy harvester (EDEH) to produce power for WTSNs.

Generally, wireless sensor nodes (WSNs) are battery operated. The lifespan of a battery is a year or two. After workable time these batteries either need recharging or replacement. However, where a large number of WSNs are applied to monitor the assets, the recharging and replacement of batteries are not feasible. Moreover, the bulky size and hazard to environment are also the concerning issues with these batteries. Harvesting energy from the ambient will have a significant impact on the field of WSNs. Energy harvesting or Energy scavenging is the technology of converting the ambient energy into electrical energy Kazmierski \& Beeby (2011). The extraction and storage of ambient energy for on-demand, off-grid use Jeremy Bickerstaffe (2011) is gaining immense attention in recent years. Energy in many different forms is available around an electrical transformer, such as in the form of thermal, mechanical vibration, RF energy and electromagnetic field. With an energy harvester one or more of these available energies can be converted into electrical energy for power requirements of WTSNs in electrical transformers. The integration of WTSN with an energy harvester can change the wireless node into a long lasting, self-powered wireless sensor unit.

Commercially available WTSNs reported in Instructables.com (2014), Veerasingam et al (2009), Gupta et al (2012) and Poonam \& Mulge (2013) can be used to monitor the temperature from -55 to $150^{\circ} \mathrm{C}$. The transmission range of the wireless temperature sensor module described in Instructables.com (2014), Veerasingam et al (2009), Gupta et al (2012) and Sensaphone remote monitoring system (2009) ranges from 10 to 90 m except in Poonam \& Mulge (2013), where a GSM module is used for monitoring of temperature. The operating voltage (from 4 to $6 \mathrm{~V}$ ) for these WTSNs is almost the same due to the usage of Arduino modules. However, the current utilization during transmission mode ranges from 30 to 1,300 mA. In sleep mode these sensor nodes consume least power; however, during transmission the overall power consumption is from 120 to $7,800 \mathrm{~mW}$.

For WTSNs at the electrical transformer, a number of ambient energies, like solar, vibration, acoustic and electromagnetic are available for harvesting. With the energy harvesters, such as vibration energy harvester Khan et al (2010) and Khan et al (2014), acoustic energy harvester Khan \& Izhar (2013), thermoelectric energy harvester Wang et al (2013) and solar energy harvester Brunelli et al (2008), the available ambient energy can be transformed into electrical energy for the utilization in WTSNs. For the electrical transformer temperature monitoring system, an electrodynamic capacitive type energy harvester reported in Chang et al (2012) is composed of an aluminum foil wrapped around the insulated power line. At a voltage of $220 \mathrm{~V}$ 
in the power line, a capacitance $(6.8 \mu \mathrm{F})$ is generated between the inner conducting wire and the wrapped aluminum foil, which is exploited to generate power. The capacitance produced in the harvester, increased proportional to the length of the foil. When an aluminum foil of 20 $\mathrm{cm}$ length is wrapped and a $2 \mu \mathrm{F}$ storage capacitor is used, the average current of $1,570 \mathrm{nA}$ is reported. However, when a $60 \mathrm{~cm}$ long aluminum foil is used, energy of about $2 \mathrm{~mJ}$ is collected in the storage capacitor in $35 \mathrm{~s}$. A current of $4.53 \mu \mathrm{A}$ is delivered to a storage capacitor of $47 \mu \mathrm{F}$ and the harvester generated an average power of $47 \mu \mathrm{W}$. The reported energy harvester successfully operated a WSN and transmitted RF data for every $42 \mathrm{~s}$. A piezoelectric energy harvester reported in Leland et al (2006) is composed of a Piezo-bimorph (T215-A4SS-103X) measuring $31.8 \times 3.2 \times 0.38 \mathrm{~mm}$. Copper foil is used to electrically connect the outer electrodes of the bimorph cantilever. Two axially poled, Neodymium $(\mathrm{NdFeB})$ disc magnets, measuring $9.5 \mathrm{~mm}$ (diameter) $\times 1.6 \mathrm{~mm}$ (thickness) are used to sandwich the free end of the bimorph. The alternating stray electromagnetic field around power cable on interaction with the magnetic field of the permanent magnets enabled the bimorph to vibrate. The resonant frequency of the harvester is tuned to $60 \mathrm{~Hz}$ in order to couple optimally with the frequency of the alternating current in the power cable. With a current of $13 \mathrm{~A}$ in power cable, and when connected to a matching load resistance of $491 \mathrm{k} \Omega$, the harvester produced a maximum power output of $345 \mu \mathrm{W}$. The AC output from the energy harvester is rectified using a full-wave bridge rectifier (DF005M) and a $1,000 \mu \mathrm{F}$ capacitor is charged with it. The capacitor is charged from 0 to $5 \mathrm{~V}$ in $196 \mathrm{~s}$. For electrical transformer monitoring system, a hybrid energy harvester reported in DeVilliers (2009) is composed of a piezoelectric type, vibration-based energy harvester and a solar-based energy harvester. In vibration-based energy harvester, a commercially available piezoelectric cantilever (PCFC-100) is used to harvest energy from the transformer vibrations. It consisted of two piezoelectric fiber composites (PFC) connected in series with a thin metallic shim in between the two PFCs layers. A copper proof mass of $3 \mathrm{~g}$ is fixed to the free end of the cantilever beam; however, the other end of the cantilever is clamped by nylon plastic bars. At an optimal load resistance of $300 \mathrm{k} \Omega$, the harvester generated a maximum output power of $897 \mu \mathrm{W}$. Moreover, after rectification the developed energy harvester charged a $1,000 \mu \mathrm{F}$ capacitor from $400 \mathrm{mV}$ to 5.12 $\mathrm{V}$ in $38 \mathrm{~s}$. However, in the hybrid energy harvester, a solar energy harvester is composed of a $114 \times 37 \times 0.2 \mathrm{~mm}$, flexible solar panel (MP3-37). The solar energy harvester produced a maximum open circuit voltage (OCV) of $3.95 \mathrm{~V}$ and when connected to a load resistance of 301.6 $\Omega$, a maximum power of $31.43 \mathrm{~mW}$ is generated. When a $1 \mathrm{~F}$, capacitor is integrated with the solar energy harvester, the capacitor is charged from $720 \mathrm{mV}$ to $3.5 \mathrm{~V}$ in about $7 \mathrm{~min}$. An electromagnetic energy harvester reported in Moghe et al (2011) is composed of an X-shaped core with copper coil wounded across it. In the harvester, the dimensions of the X-shaped open core are $54 \times 64 \times 18 \mathrm{~mm}$ and the wound coil is made up of 300 turns. When the developed harvester is attached along the power cable, it produced a power of $90 \mathrm{~mW}$ at $800 \mathrm{~A}$ current in the cable. At a cable current of $60 \mathrm{~A}$, an open circuit AC voltage of $200 \mathrm{mV}$ is obtained with the harvester. AC to DC boost circuit is used to rectify the voltage from the harvester and is supplied to charge a super capacitor for wireless sensing system. The electromagnetic energy harvester Bhuiyan et al (2010) consisted of a multi-turn coil wounded around a magnetic core material. Several layers of high permeability mu-metal are used to fabricate the magnetic core material. A thin wire is wrapped around the cylindrical core in such a way that the wire is parallel to the power cable axis. The developed energy harvester is composed of five layers of mu-metal and 280 turns wound coil. When a power cable is inserted through the wound coil aperture and a maximum current of $13.5 \mathrm{~A}$ is passed through a power cable, the harvester produced an $\mathrm{AC}$ voltage of $0.88 \mathrm{~V}$. Moreover, for the same cable current, a maximum power of $10.385 \mathrm{~mW}$ is 
obtained at matching impedance of $76.1 \Omega$. However, when the harvester is connected to a rectifier, the voltage boosted up to $2.4 \mathrm{~V} \mathrm{DC}$, which is used to charge a rechargeable battery $(1.2 \mathrm{~V})$ from zero to $1.2 \mathrm{~V}$ in $180 \mathrm{~min}$. An electromagnetic energy harvester composed of I-shaped steel core and 200 turns of copper wound coil is developed by Moghe et al (2010). The harvester is clamped with a power cable. At a cable current of $50 \mathrm{~A}$ the energy harvester generated an OCV of $0.2 \mathrm{~V}$. After rectification the voltage is boosted from $0.2 \mathrm{~V}$ AC to $3.3 \mathrm{~V} \mathrm{DC}$ which is then stored into a $1 \mathrm{~F}$ ultra-capacitor. An electromagnetic energy harvester developed and reported in Lee (2012) is composed of a core and wound coil. In the harvester the core is made by gluing together 0.014 inch thick rectangular pieces of the metal (mu-metal). Thin copper wire is used to produce a 500 turns wound coil. With a 12 A cable current, the harvester produced $470 \mathrm{mV}$ RMS voltage and a maximum power of $7 \mathrm{~mW}$ at matching impedance $(25 \Omega)$. Cockcroft-Walton rectifier is used to convert and step up the generated AC voltage into DC. After rectification an open circuit rectified voltage of $15 \mathrm{~V} \mathrm{DC}$ is reported for the harvester.

\section{Modeling and simulation}

EDEH presented in this paper consists of a copper wound coil, a mild steel cylinder (core) and a Teflon housing (to safe guard the harvester's core and wound coil from the envirnomental effects, such as rain, sunlight, fog and humidity). The cross-sectional and exploded views of the EDEH are shown in figure 1 and figure 2 respectively. The split-cylindrical design for the harvester's core and housing permits the EDEH to be directly wrapped around the power cable without shutting down the power supply or without disconnecting the power cable from the transformer. The inner radius $r_{1}$ of the cylinderical core of the energy harvester depends on the radius of the power cable. The radius $r_{1}$ needs to be adjusted such that the coil sees high magnetic flux density. In power cables, such as shown in figure 3 , the magnetic flux density is directly proportional to the current flowing through it and inversely proportional to the distance from the center of the cable Sordiashie (2012).

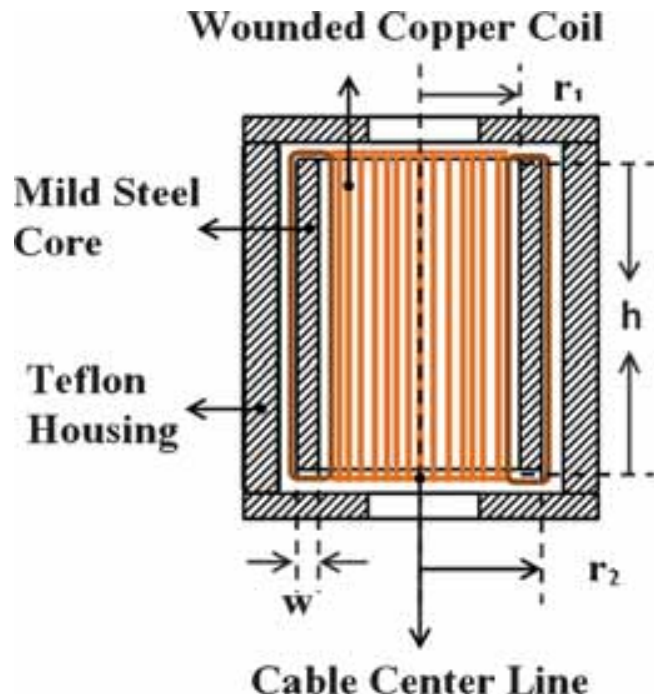

Figure 1. Cross-sectional view of electrodynamic energy harvester. 


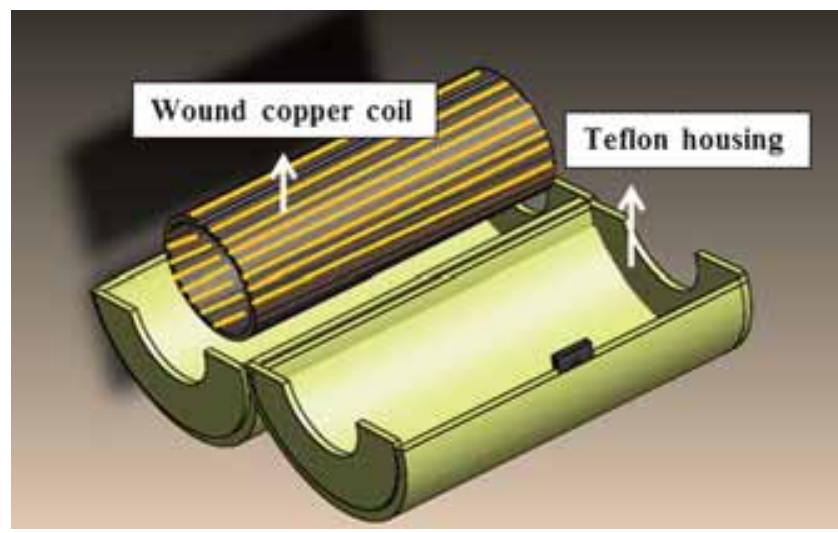

Figure 2. Exploded view of electrodynamic energy harvester.

The power cable shown in figure 3 is a single core cable composed of 19 copper wires and two layers of insulated material. The outer diameter of the cable is $20 \mathrm{~mm}$. In order to visualize the magnetic flux density around a power cable (figure 3) modelling and simulation for the cable is performed using COMSOL Multiphysics. Figure 4 shows the magnetic flux density around a single core insulated cable. The simulation is performed for a current of $50 \mathrm{~A}$ in the power cable and the magnetic flux density distribution along the cable's cross-sectional plane is shown in figure 4. It is evident from the simulation that the magnetic flux density is mostly concentrated at a radial distance of about $0.5 \mathrm{~cm}$ from the center of the cable. This radial distance is actually inside the cable's insulation and therefore due to the presence of insulation this maximum magnetic flux density cannot be utilized for energy harvesting. The magnetic flux density that can be beneficial for harvesting actually is available after insulation, which is $1 \mathrm{~cm}$ radial distance from the center of the cable.

Figure 5 shows the simulation results for the magnetic flux density obtained along line $\mathrm{K}$ in figure 4. The simulations are performed for cable current of 27, 72 and $155 \mathrm{~A}$. As the cable current increases the magnetic flux density around the cable also increases. At all current levels,

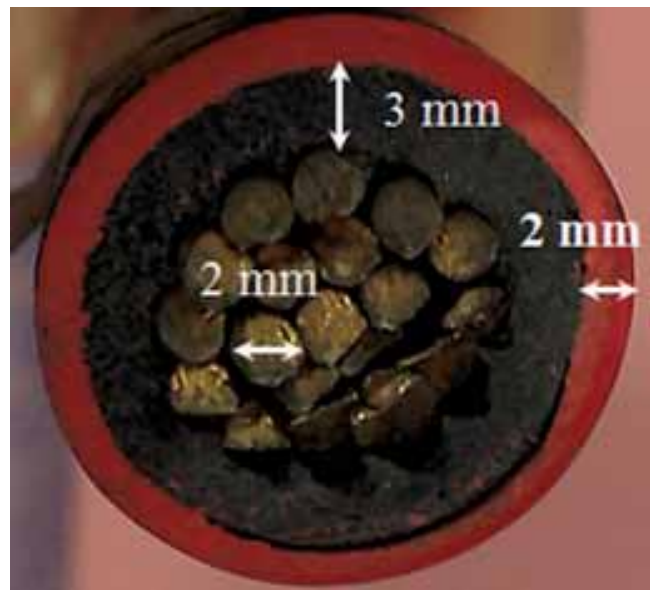

Figure 3. Photographic image of a single core power cable. 


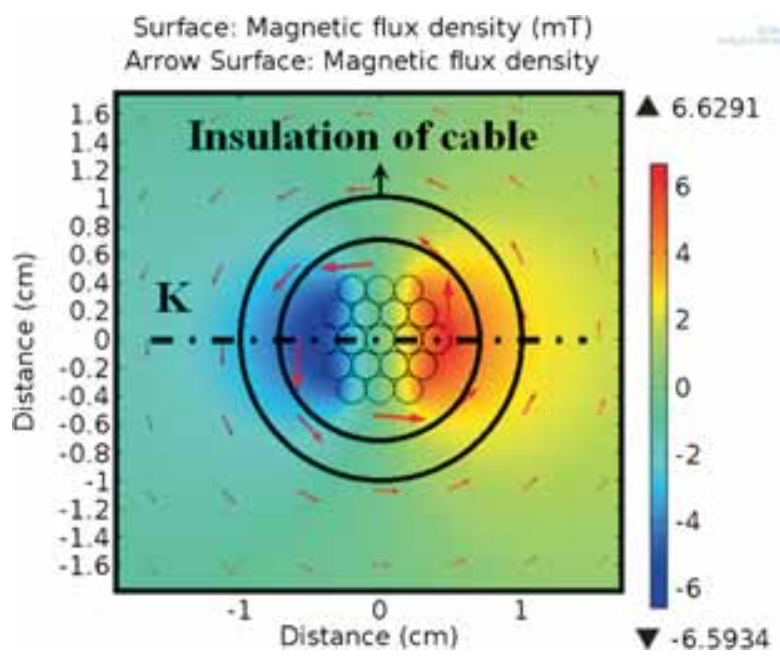

Figure 4. Magnetic flux density distribution in a single core cable at $50 \mathrm{~A}$ current in the cable.

magnetic flux density reached to it maximum value at a radial distance of about $0.5 \mathrm{~cm}$ (inside the insulation) and then it gradually decreases. At the radial distance of $1 \mathrm{~cm}$, magnetic flux densities of $0.55,1.44$ and $3.1 \mathrm{mT}$ are available (along the line $\mathrm{M}$ in figure 5) at current levels of 27,72 and 155 A respectively. However, the magnetic flux density reduced to $0.36,1$ and $2.1 \mathrm{mT}$ at the radial distance of $1.5 \mathrm{~cm}$. The distribution of magnetic flux density lines around the cable at a current level of $155 \mathrm{~A}$ is shown in figure 6 . The simulation results in figure 4 and figure 5 show that from the cable's center, the radial distance from $1 \mathrm{~cm}$ to $1.5 \mathrm{~cm}$ is the most feasible dimension for the wound coil of the proposed energy harvester.

For EDEH (figure 1), the induced OCV

$$
V(t)=\frac{-N d \phi(t)}{\mathrm{d} t}
$$

obtained by Faraday's law Jay (2008) depends on the number of coil turns $N$ and the magnetic flux $\phi$.

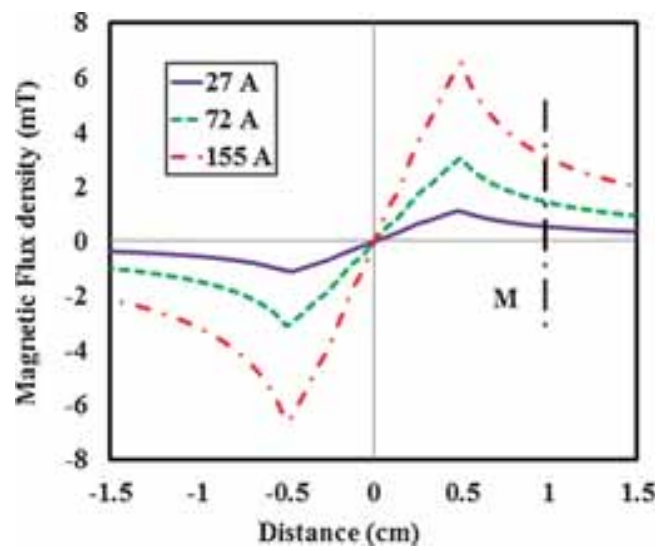

Figure 5. Magnetic flux density at cable current of 27, 72 and $155 \mathrm{~A}$ and along line $\mathrm{K}$ in figure 4. 


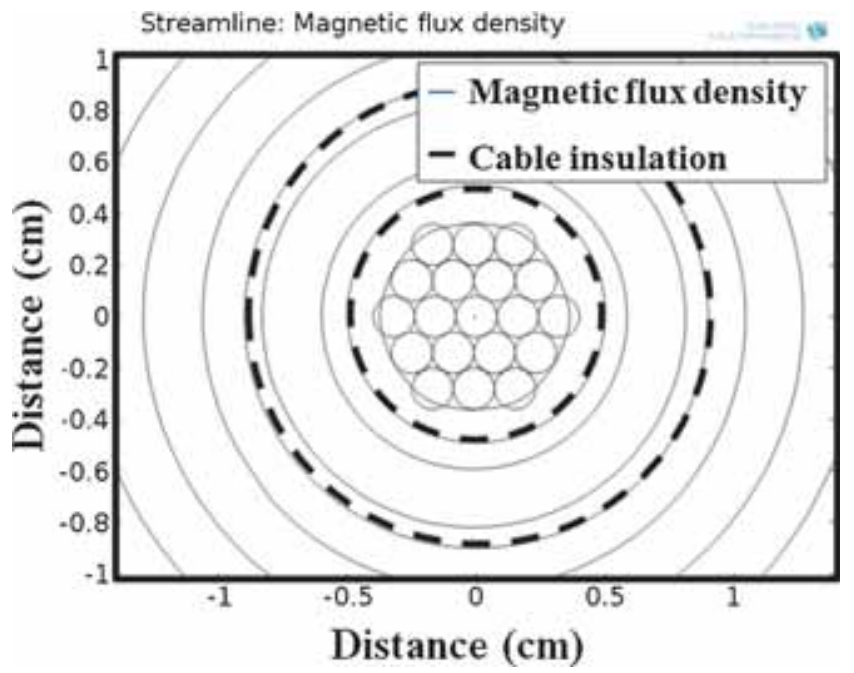

Figure 6. Magnetic flux density lines around a single core power cable at a current level of $155 \mathrm{~A}$.

Through the harvester's coil, the magnetic flux

$$
\phi=\int B \cdot \mathrm{d} A=\int B \mathrm{~d} A
$$

can be obtained with the magnetic flux density $B$ over the coil area $A$.

By substituting (2) in (1), leads to a form

$$
V(t)=\frac{-N d \phi(t)}{\mathrm{d} t}=-N \int \frac{\mathrm{d} B(t)}{\mathrm{d} t} \mathrm{~d} A
$$

Around the current carring power cable, the magnetic flux density Chang et al (2012)

$$
B(t)=\frac{\mu I(t)}{4 \pi r}
$$

is inversely proportional to the distance $r$ from the centerline of the cable and also its strength depends on the current $I(t)$ passing through the cable and the magentic permebailty $\mu$ of the core material.

Due to the small radial distance $\mathrm{d} r$ from the center of the cable, the small variation

$$
\mathrm{d} B(t)=\frac{-\mu I(t)}{4 \pi} \frac{1}{r^{2}} \mathrm{~d} r
$$

in the magnetic flux density can be used to obtain the magnetic flux density

$$
B(t)=\frac{-\mu I(t)}{4 \pi} \int_{r^{1}}^{r^{2}} \frac{1}{r^{2}} \mathrm{~d} r
$$

over the single loop of the wound coil, whose vertical sides are at a distance $r_{1}$ and $r_{2}$ from the centerline of the cable as shown in figure 1. Where $r_{1}$ is the radial distance from the center of 
the cable to the inner side of copper coil and $r_{2}$ is the radial distance between the cable's center and the outer end copper coil.

By differentiating (6) with respect to time, deduce Eq. (6) for the rate of change of the magnetic flux density

$$
\frac{\mathrm{d} B(t)}{\mathrm{d} t}=\frac{\mu}{4 \pi} \int_{r_{1}}^{r_{2}} \frac{1}{r^{2}} \mathrm{~d} r \frac{\mathrm{d} I(t)}{\mathrm{d} t}
$$

around the cable and into the single loop of the coil.

The infinitesimal area

$$
\mathrm{d} A=h \mathrm{~d} r
$$

of the coil's single loop of height $h$ is integrated over the range $r_{1}$ and $r_{2}$ to obtain the whole loop area

$$
A=h \int_{r^{1}}^{r^{2}} \mathrm{~d} r
$$

of the single loop.

By substititing (7) and (9) in (3), the induced voltage

$$
V(t)=-N \frac{\mu}{4 \pi} h \frac{\mathrm{d} I(t)}{\mathrm{d} t} \int_{r_{1}}^{r_{2}} \int_{r_{1}}^{r_{2}} \frac{1}{r^{2}} \mathrm{~d} r \mathrm{~d} r
$$

in $N$ turns of the coil is obtained. The alternating current $I(t)=I_{o} \sin (\omega t)$ in the power cable can be used to substitute the rate of change of current

$$
\frac{\mathrm{d} I}{\mathrm{~d} t}=\omega I_{o} \cos (\omega t)
$$

in Eq. (10) to obtain the voltage generation

$$
V(t)=-N \omega I_{0} \frac{\mu}{4 \pi} h \frac{\left(r_{2}-r_{1}\right)^{2}}{r_{2} r_{1}} \cos (\omega t)=-N \omega I_{0} \frac{\mu}{4 \pi} h \frac{w^{2}}{r_{2} r_{1}} \cos (\omega t)
$$

in the harvester's coil in terms of the coil width $w=r_{2}-r_{1}$, frequency $\omega$ and amplitude $I_{0}$ of the current flowing through the cable.

When the load resistance $R_{\mathrm{L}}$ is connected to the harvester's coil with resistance $R_{\mathrm{C}}$, the amplitude of load voltage

$$
V_{\mathrm{L}}=\frac{R_{\mathrm{L}}}{R_{\mathrm{L}}+R_{\mathrm{C}}} N \omega I_{\mathrm{o}} \frac{\mu}{4 \pi} h \frac{w^{2}}{r_{1}\left(w+r_{1}\right)}
$$

can be used to compute the RMS voltage

$$
V_{\mathrm{rms}}=\frac{R_{\mathrm{L}}}{\sqrt{2}\left(R_{\mathrm{L}}+R_{\mathrm{C}}\right)} N \omega I_{\mathrm{o}} \frac{\mu}{4 \pi} h \frac{w^{2}}{r_{1}\left(w+r_{1}\right)}
$$

and the average power

$$
P_{\mathrm{L}}=\frac{V_{\mathrm{rms}}^{2}}{R_{\mathrm{L}}}=\frac{1}{2} \frac{R_{\mathrm{L}}}{\left(R_{\mathrm{L}}+R_{\mathrm{C}}\right)^{2}}\left[N \omega I_{\mathrm{o}} \frac{\mu}{4 \pi} h \frac{w^{2}}{r_{1}\left(w+r_{1}\right)}\right]^{2}
$$

dissipated in the load resistance $R_{\mathrm{L}}$. 


\section{Fabrication of prototype}

The fabrication steps of the developed EDEH in this work are shown in figure 7. The inductive energy harvester consists of a mild steel core with a copper wire wrapped around to form a copper wound coil. The copper wound coil is enclosed in a Teflon cavity. Mild steel sheet is used as the core material for the energy harvester, figure 7(a). Copper wire of diameter $180 \mu \mathrm{m}$, is wound over the mild steel sheet for producing the wound coil, figure 7(b). The steel sheet, carrying the copper wire is then bent around a power cable to produce the cylindrical shaped, 800 turns wound coil for the harvester, figure 7(c). The Teflon housing for the

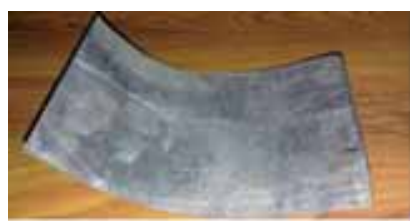

(a)

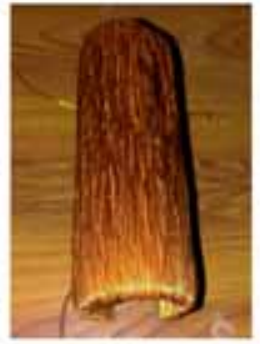

(c)

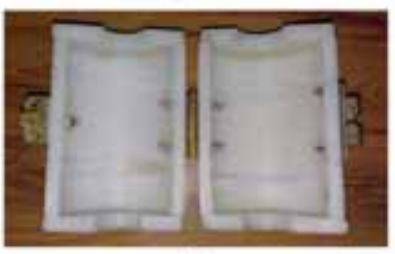

(e)

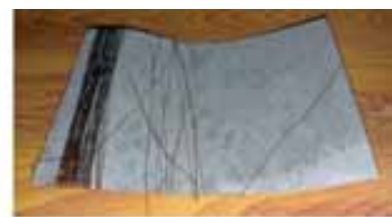

(b)

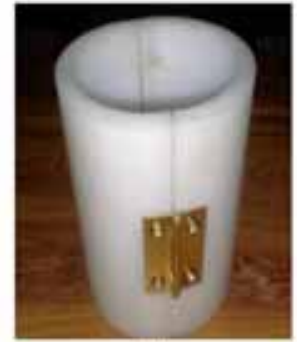

(d)

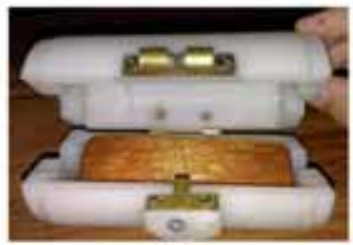

(f)

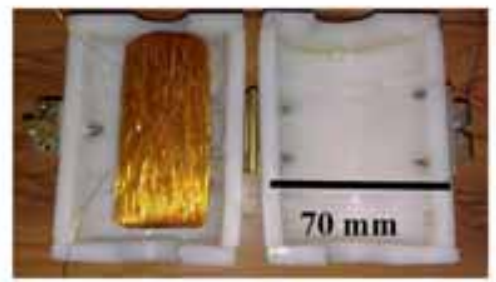

(g)

Figure 7. Photographic images of the developed prototype EDEH: (a) mild steel plate used as a core material, (b) initial wrapping of copper wire around the core material, (c) wound coil and core bent in cylindrical shape (d) hinged split Teflon cylinder for harvester's housing, (e) Complete Teflon housing for the energy harvester, (f) locking system in housing for prototype, (g) complete prototype having the inductive energy harvester. 
Table 1. Dimensions and parameters of the developed EDEH prototype.

\begin{tabular}{lcc}
\hline Harvester main part & Description & Value \\
\hline Core & Material & Mild steel \\
& Height & $8.09 \mathrm{~cm}$ \\
& Diameter & $6.9 \mathrm{~cm}$ \\
Housing & Thickness & $1.2 \mathrm{~mm}$ \\
& Material & Teflon \\
& Height & $10.7 \mathrm{~cm}$ \\
Wound coil & Outer diameter & $9 \mathrm{~cm}$ \\
& Inner diameter & $2 \mathrm{~cm}$ \\
& Material & Copper \\
& Wire diameter & $180 \mu \mathrm{m}$ \\
& Number of turns & 800 \\
& Resistance & $24 \Omega$ \\
& Overall thickness & $1.2 \mathrm{~mm}$ \\
\hline
\end{tabular}

energy harvester is fabricated from the commercially available Teflon cylindrical block. First a hollow cylinder is made using a conventional machining, the hollow cylinder is then cut in half (long the length) and the two cut pieces are then joined together with a hinge, which enables the housing to open and close around the power cable, figure 7(d). Holes are drilled into the Teflon disks and are cut in halves and then bonded with epoxy to the open ends of the hollow cylinder, figure 7(e). A locking mechanism, figure 7(e), is also introduced in the harvester casing to firmly keep the energy harvester around the power cable. Finally the cylindrical wound coil is then place in the Teflon housing to develop the complete EDEH, figure $7(\mathrm{~g})$. The dimensions and parameters of the developed EDEH prototype are listed in table 1 .

\section{Characterization of the developed EDEH}

Figure 8(a) shows the block diagram of the experimental setup for the characterization of the developed prototype. The EDEH is wrapped around the output power cable of electrical transformer carrying high current. The output terminals of the harvester are connected to a load resistance and a digital multi-meter (DMM) is connected in parallel to measure the voltage drop across the load. Moreover, the same DMM is used to measure the current flowing in the harvester's coil under variable load resistance. Ammeter (Digital clamp meter, type: DM6266) is utilized to measure the current flowing in the power cable. Figure $8(\mathrm{~b})$ shows the photograph obtained during the characterization of the EDEH at the site.

The RMS load voltage as a function of load resistance at different cable current levels (27 A, $72 \mathrm{~A}$ and $155 \mathrm{~A}$ ) is shown in figure 9. The simulation result is obtained with Eq. (13). The simulation of the analytical model (Eq. 13) quiet agrees with the experimental results. The slight deviation of the model from the experimental data is due to the variation in the current flowing through the power cable, cable's diameter non-uniformity and fabrication uncertainty of the energy harvester. At higher current level in the power cable, more voltage is generated by the harvester which is attributed to the stronger magnetic field generation 


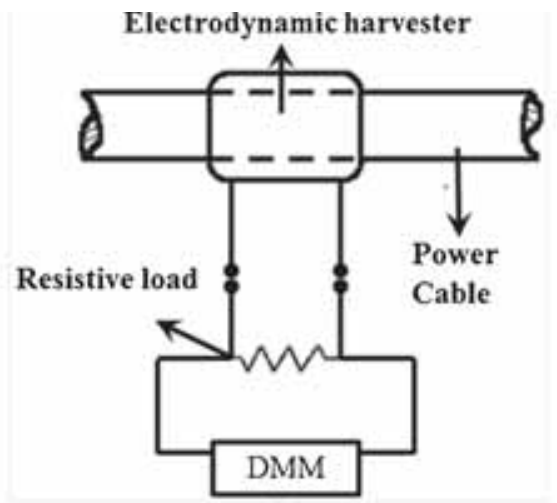

(a)

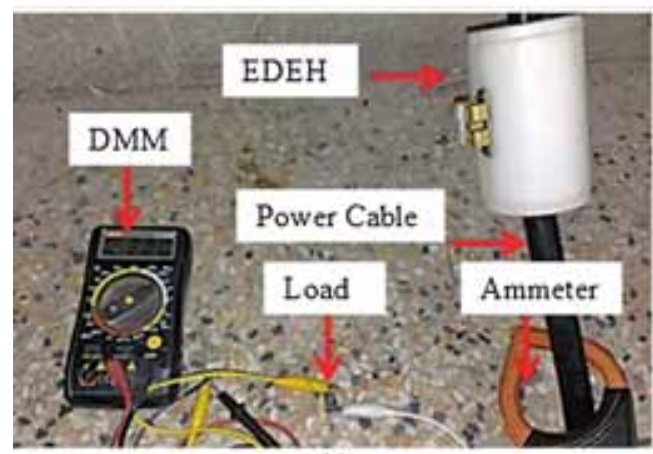

(b)

Figure 8. Characterization of the developed EDEH: (a) Block diagram of the experimental setup, (b) photograph of EDEH during experimentation.

around the power cable. As the load resistance is increased, less current is allowed by the resistor to flow and in turn the load voltage increases. At a load resistance of $100 \Omega$, maximum load voltages of $0.356,1.09$ and $2.58 \mathrm{~V}$ are produced under 27, 72 and $155 \mathrm{~A}$ cable currents respectively.

The average load power as a function of the load resistance at current levels of 27, 72 and $155 \mathrm{~A}$ is shown in figure 10. Equation (14) is used for the computation of simulation results at these current levels. The analytical model (Eq. 14) is in agreement with the experimental results. The slight deviation of the simulation from the experimentation is due to the electrical current variation in the power cable and dimensional uncertainty of power cable and harvester. From the harvester, the maximum power is generated around $24 \Omega$ load resistance which is actually the resistance of the coil. Under optimum load condition, the EDEH produced the maximum power of 2.99, 19.66 and $112.03 \mathrm{~mW}$ for a cable current of 27,72 and $155 \mathrm{~A}$ respectively.

In the developed EDEH, the induced voltage is alternating (AC); however, DC voltage is required for the operation of WTSNs. Normally, a rectifier is used to convert the voltage from AC to DC. Figure 11(a) shows the block diagram of the experimental setup for the DC 


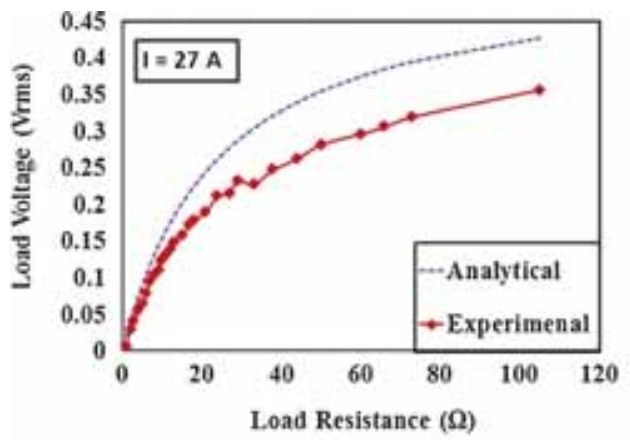

(a)

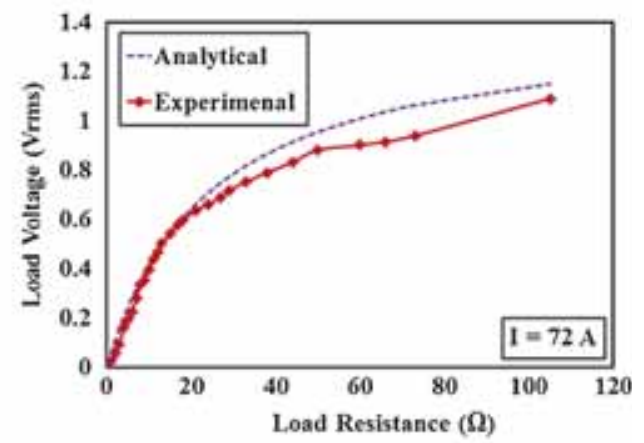

(b)

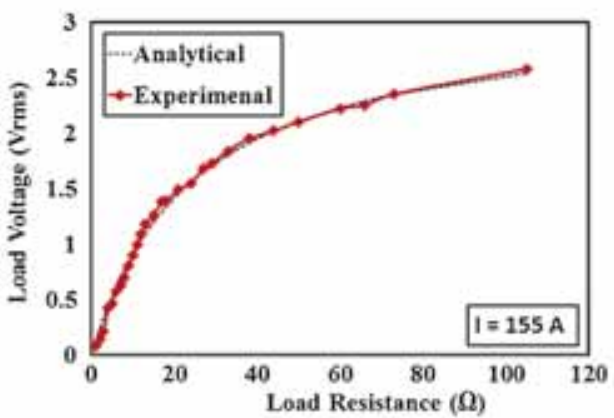

(c)

Figure 9. Load voltage as a function of load resistance at different cable current levels: (a) load voltage versus load resistance at $27 \mathrm{~A}$ cable current, (b) load voltage versus load resistance at $72 \mathrm{~A}$ cable current, (c) load voltage versus load resistance at $155 \mathrm{~A}$ cable current.

voltage measurement, when a rectifier is connected to the developed EDEH. The wound copper coil is wrapped around the power cable carrying the electrical current, the output terminals of the harvester are connected to the 3-stage Cockcroft-Walton rectifying circuit, afterwards it is connected to the load resistance and a DMM is connected in parallel to measure the voltage drop across the load resistor. Figure 11(b) shows the photographic image during the DC voltage measurement from the energy harvester. 


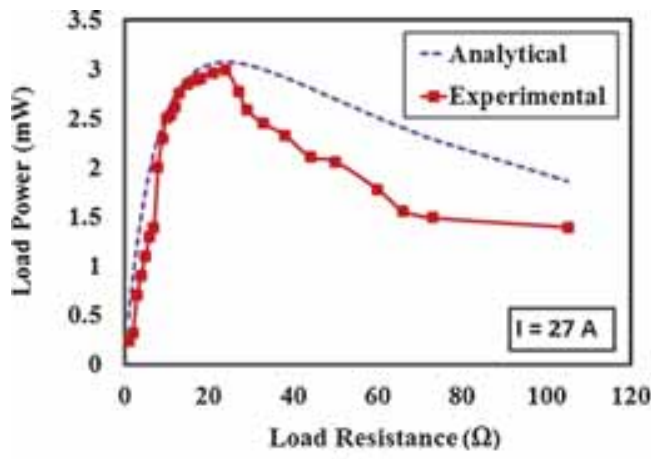

(a)

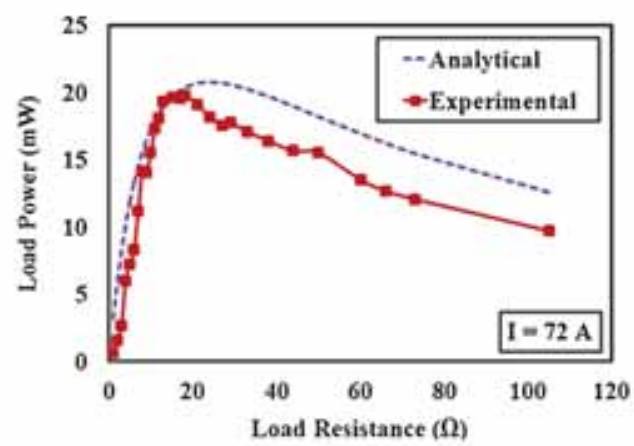

(b)

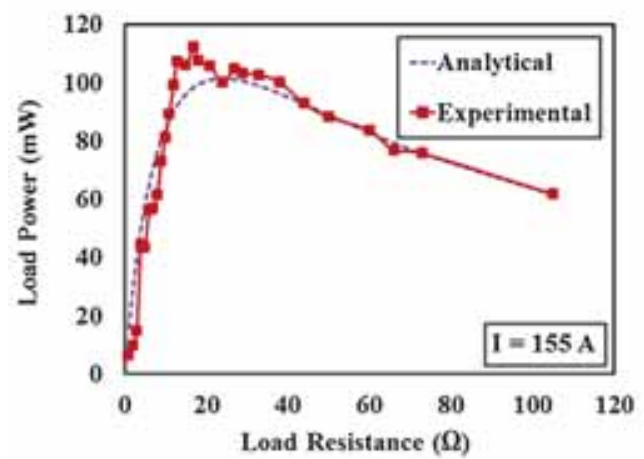

(c)

Figure 10. Average power dissipated as a function of load resistance at different cable currents: (a) load power versus load resistance at 27 A cable current, (b) load power versus load resistance at $72 \mathrm{~A}$ cable current, (c) load power versus load resistance at 155 A cable current.

Figure 12 shows the DC voltage drop in the load for cable currents of 63 and 93 A. Load voltage increases across the load resistor with increase in resistance. Figure 13 shows the load power for cable current of 63 and 93 A. For figure 13, power is computed as a product of measured voltage and current. At both current levels, the power is maximum at a load resistance of about $185 \Omega$. An optimum power of 9 and $19 \mathrm{~mW}$ are generated at a cable current of 63 and 93 A respectively. Due to the integration of the rectifying circuit with the energy 


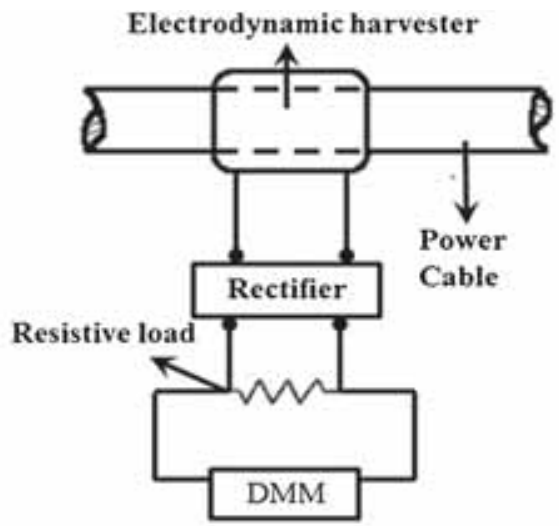

(a)

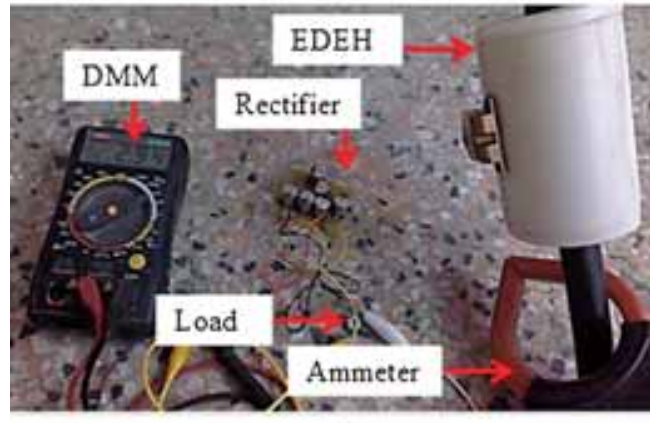

(b)

Figure 11. Experimental setup for DC voltage measurement from EDEH: (a) Block diagram of the experimental setup, (b) photographic image during the DC voltage measurements.

harvester, the optimum load condition shifts from $24 \Omega$ to $185 \Omega$, which is attributed as a result of the inclusion of the rectifier circuit impedance in the overall circuitry. Moreover, due to the power consumption of the rectifier circuit, comparatively a slight reduction in power has been observed.

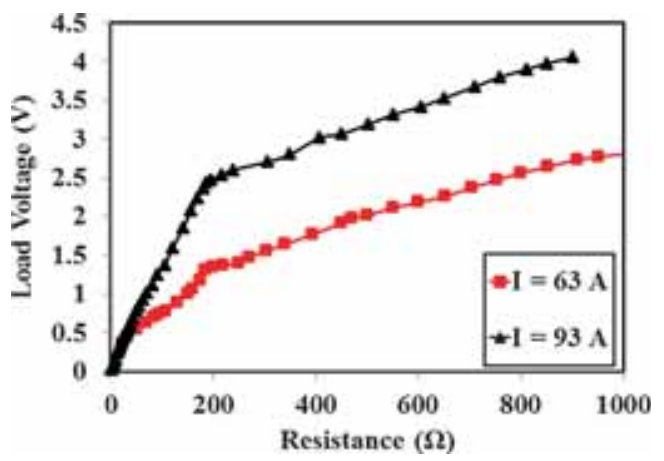

Figure 12. Load DC voltage versus load resistance. 


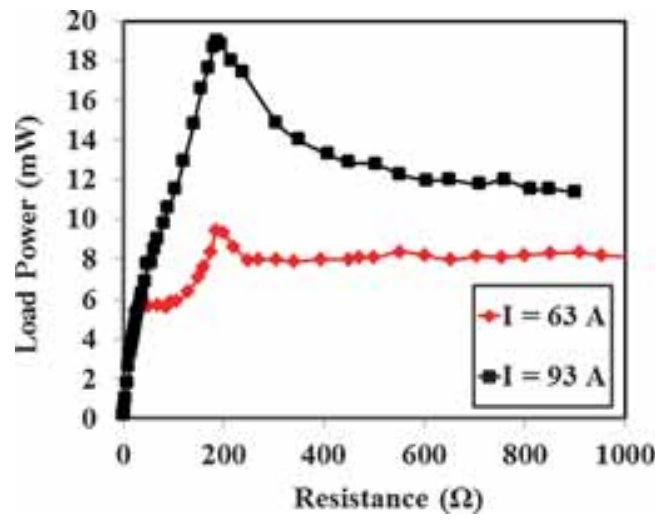

Figure 13. Load power versus load resistance.

\section{Generated power storage}

In WSNs, after the voltage rectification, the power is either utilized for the operations or in situation of surplus power generation; it is stored in a super capacitor or battery. Figure 14 shows the charging of $3.8 \mathrm{~V}$, Nickel-Cadmium $(\mathrm{Ni}-\mathrm{Cd})$ rechargeable battery, when it is connected to the energy harvester after the rectifying circuit. The battery with initial charge of $1 \mathrm{~V}$ started charging slowly and gradually. In the first 40 min the battery was charging comparatively faster; however,
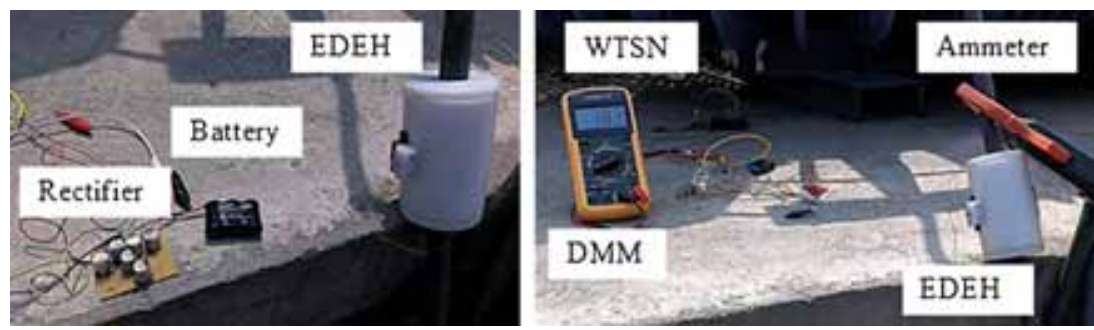

Figure 14. Photographic image of charging of battery with the developed EDEH.

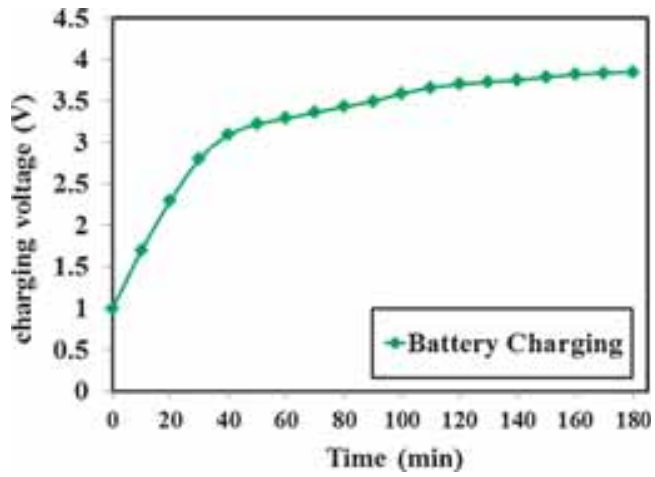

Figure 15. Battery's voltage as a function of time during charging with the EDEH. 
as the charge build up, the battery started charging slowly. In about $180 \mathrm{~min}$ the battery gets fully charged up to the maximum voltage, which is recorded as $3.85 \mathrm{~V}$ and shown in figure 15.

In this work, a WTSN used for the electrical transformer temperature monitoring is composed of LM35, temperature sensor, nRF24L01, transceiver and an Arduino mini microcontroller. The power consumption for WTSN is about $108 \mathrm{~mW}$ during transmission, which is greater in comparison to the maximum power generated by the developed EDEH. For operation of the WTSN, a rechargeable battery is used. A Battery, which is charged in almost $180 \mathrm{~min}$ from $1 \mathrm{~V}$ to $3.85 \mathrm{~V}$ with the EDEH, is connected to the WTSN. Rechargeable battery operates the WTSN for more than $24 \mathrm{~h}$. After this time the voltage across the battery drops to $3.3 \mathrm{~V}$ and data transmission to the receiver is terminated. However, the EDEH needs only 40 min to completely recharge the battery again. In the temperature monitoring system these $40 \mathrm{~min}$ could be the delay or sleep mode and the system will keep monitoring the temperature of the electrical transformer with only a break of $40 \mathrm{~min}$ in a day. Figure 16 shows the complete setup of the system, during monitoring of the transformer's temperature. A temperature sensor (LM35) was attached with the transformer's oil

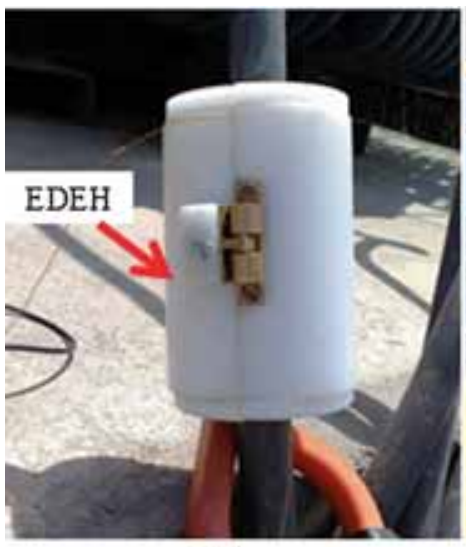

(a)

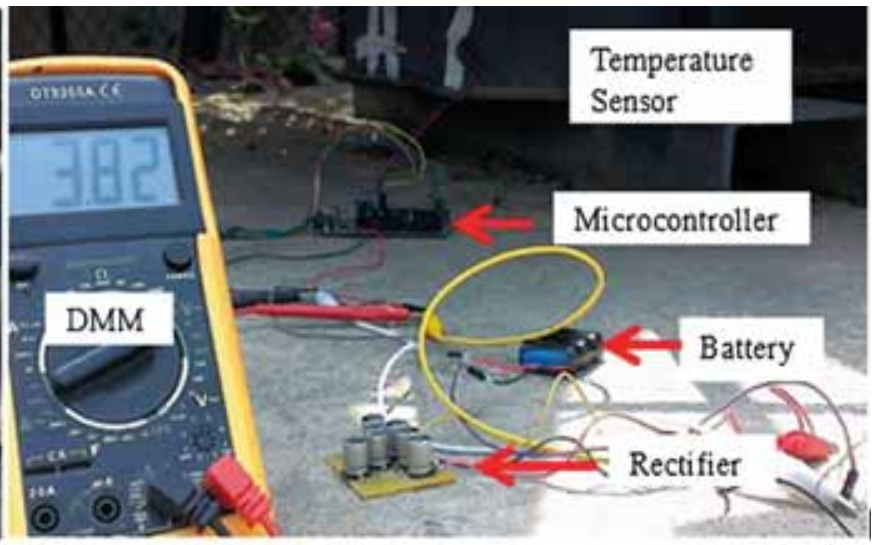

(b)

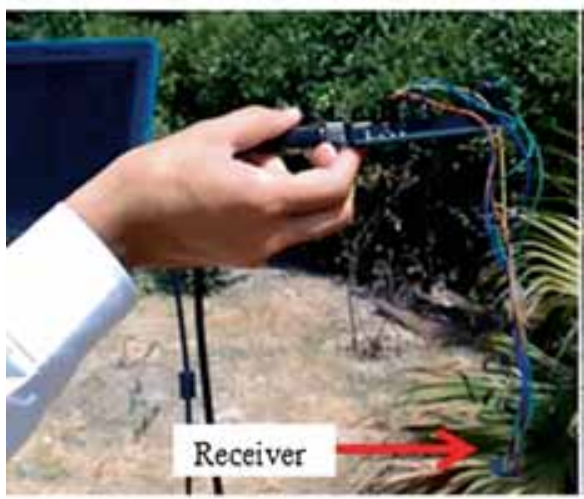

(c)

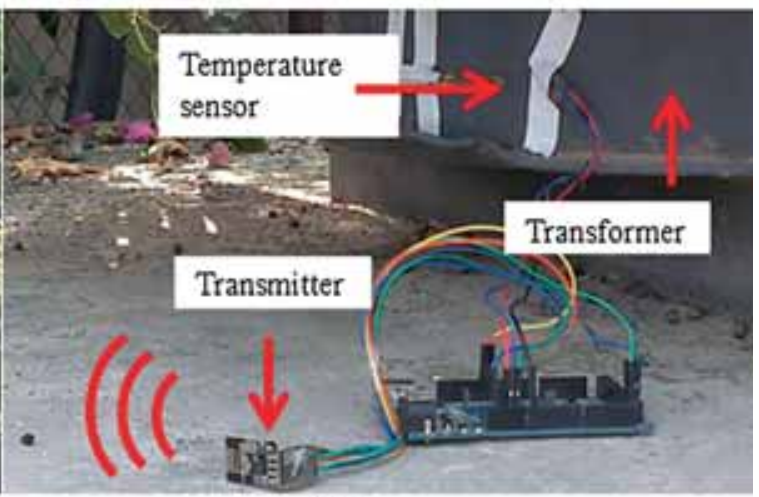

(d)

Figure 16. Photographic image of autonomous wireless temperature system: (a) the developed EDEH on power cable, (b) the circuitry connected with EDEH and temperature sensor connected to the transformer, (c) wireless temperature sensor node, $(\mathbf{d})$ receiver connected with laptop and monitoring the temperature of transformer. 


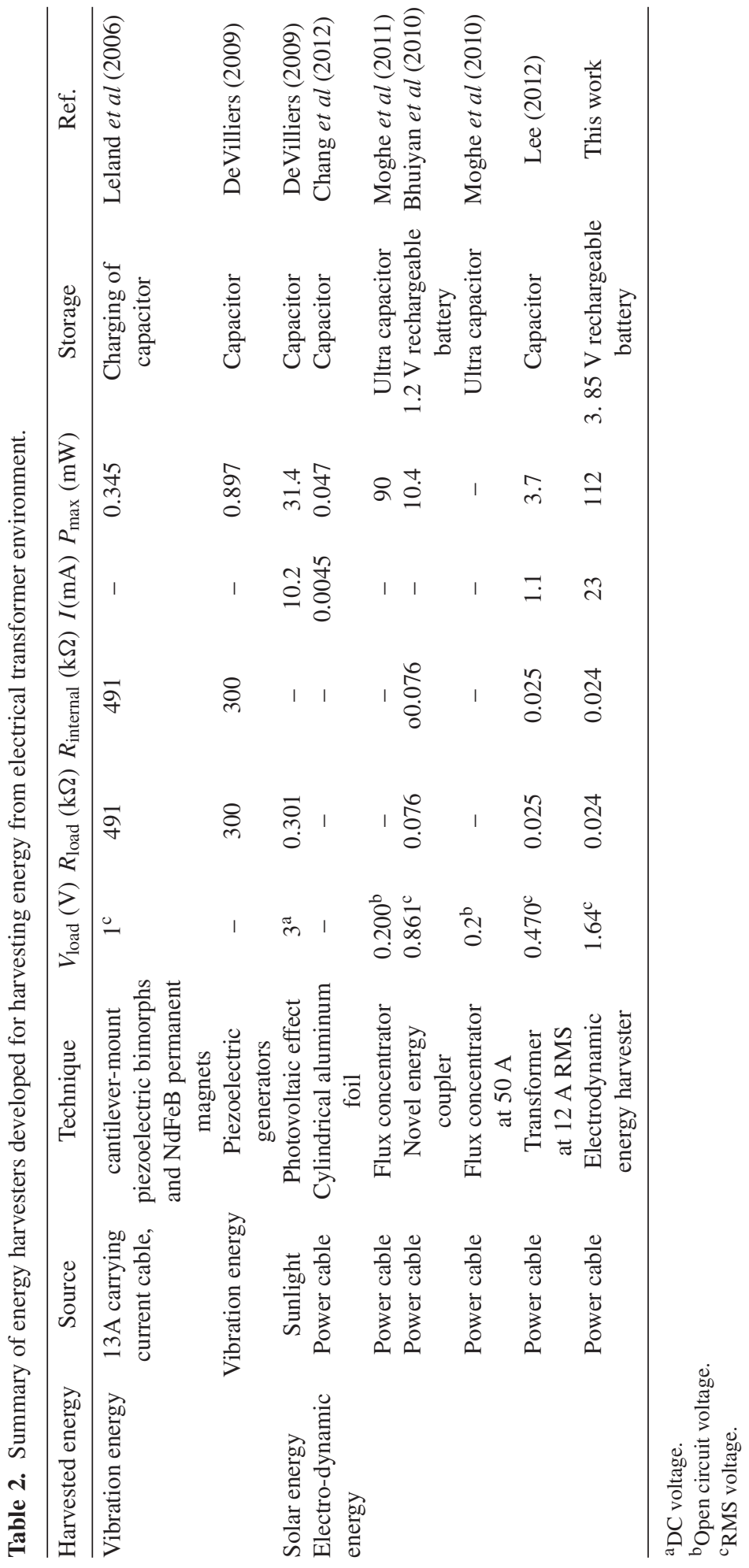


tank externally. The temperature of transformer tank was $41^{\circ} \mathrm{C}$ when monitored with the devised autonomous WTSN. For measuring the internal oil temperature inside a transformer, a temperature sensor (DS18B20) reported in Rong \& Zuozuo (2005) and Zhao et al (2013) can be used. For this purpose the DS18B20 sensor has to be mounted in a drilled sealed hole and connected to the same circuit components as mentioned in case of LM35 temperature sensor.

Energy harvesters that are developed for harvesting energy from the electrical transformer environment and reported in literature are listed in table 2.

Vibration based energy harvesters Leland et al (2006), DeVilliers (2009), those converted the transformer vibration into electrical energy produced a maximum power of $0.897 \mathrm{~mW}$. However, the solar energy harvester De Villiers (2009) generated a DC voltage of $3 \mathrm{~V}$ and a power of $31.4 \mathrm{~mW}$. EDEHs Chang et al (2012), Moghe et al (2011), Bhuiyan et al (2010), Moghe et al (2010), Lee (2012) and the harvester this work produced high voltages in comparison to the other developed energy harvesters. Moreover, the power produced by the EDEH developed in this work is higher than that of all other reported energy harvesters. The voltage generated by these energy harvesters is the range from 0.2 to $3 \mathrm{~V}$. The maximum voltage of $3 \mathrm{~V}$ is produced by the energy harvester reported in DeVilliers (2009). However, among the EDEHs, the energy harvester developed in this work generated the maximum voltage $(1.64 \mathrm{~V})$. The internal impedance of the harvester DeVilliers (2009) is very high $(300 \mathrm{k} \Omega)$ in comparison. The EDEH reported in this work has the lowest internal impedance, which is only $24 \Omega$. Moreover, all the developed energy harvesters are reported to be capable of charging either a capacitor or rechargeable battery.

\section{Conclusion}

For temperature monitoring of electrical transformers, an EDEH is developed to operate a WTSN. The reported EDEH is composed of a mild steel core, a copper wound coil of 800 turns and a Teflon housing of $8.5 \mathrm{~cm}$ height. Due to the split-cylindrical design, the harvester can be wrapped very easily around the output power cable of the electrical transformer without shuttingoff the power or disconnecting the power cable. For the architecture of the EDEH an analytical model for the voltage generation is also devised. The EDEH is characterized on site at different current levels passing through the output cable of an electrical transformer. A maximum RMS voltage of $2.58 \mathrm{~V}$ and an optimum average power of $112.03 \mathrm{~mW}$ are produced by the developed energy harvester from the stray magnetic field around the cable. A 3-stage Cockcroft-Walton rectifying circuit is used to convert the harvester's AC output voltage into DC voltage. With the rectified DC voltage, a $3.8 \mathrm{~V}$ rechargeable battery (Nickel-Cadmium) is charged and a WTSN is successfully operated for monitoring the temperature of an electrical transformer. The power produced by the developed EDEH is comparable and actually better than the other reported EDEHs. Moreover, the EDEH is quiet capable of operating a WTSN for the temperature monitoring of electrical transformers.

\section{References}

Bhuiyan R, Dougal R and Ali M 2010 A miniature energy harvesting device for wireless sensors in electric power system. IEEE Sensors J. 10: 1249-1258

Brunelli D, Benini L, Moser C and Thiele L 2008 An efficient solar energy harvester for wireless sensor nodes. Proceedings of Design, Automation and Test in Europe (DATE '08) pp. 104-109. doi: 10.1109/DATE.2008.4484670 
Chang K, Kang S, Park K, Shin S, Kim H-S and Kim H 2012 Electric field energy harvesting powered wireless sensors for smart grid. J. Electr. Eng. Technol. 7: 75-80

DeVilliers D J 2009 Hybrid energy harvester system for a condition monitoring mote. MSc dissertation, Cape Peninsula University of Technology, Cape Town, South Africa

Gupta S, Mitra N and Kumar A 2012 X-bee based temperature monitoring. VSRD Int. J. Electr. Electron. Commun. Eng. 2: 412-416

Instructables.com 2014 LOG wireless temperature monitoring [online]. Available from http://www. instructables.com/id/LOG-Wireless-Temperature-Monitoring/ [Accessed 2 October 2014]

Jay N 2008 Physics of life sciences. Springer, New York, USA

Jeremy Bickerstaffe 2011 Energy harvesting. A white paper published by Sagentia [online]. Available from http://www.sagentia.com/resources/white-papers/2011/energy-harvesting.aspx. [Accessed 28 June 2014]

Kazmierski T and Beeby S 2011 Energy harvesting systems: principles, modeling and applications. Springer, New York, USA

Kennedy S P and Gordner T 2013 Hot spot studies for sheet wound transformer windings [online]. Available from http://www.qualitrolcorp.com/uploadedFiles/Siteroot/Products/ Hot_Spot_Studies_for_Sheet_Wound_Transformer_Windings.pdf. [Accessed 28 June 2014]

Khan F, Farrokh Sassani F and Stoeber B 2010 Copper foil-type vibration-based electromagnetic energy harvester. J. Micromech. Microeng. 20: 125006. doi: 10.1088/0960-1317/20/12/125006

Khan F, Farrokh Sassani F and Stoeber B 2014 Nonlinear behaviour of membrane type electromagnetic energy harvester under harmonic and random vibrations. Microsyst. Technol. 20: 1323-1335 doi: 10.1007/s00542-013-1938-1

Khan F U and Izhar 2013 Acoustic-based electrodynamic energy harvester for wireless sensor nodes application. Int. J. Mater. Sci. Eng. 1: 72-78

Lee V C 2012 Energy harvesting for wireless sensor networks. MSc dissertation, University of California, Berkeley, USA

Leland E, White R and Wright P 2006 Energy scavenging power sources for household electrical monitoring. The $6^{\text {th }}$ international workshop on micro and nanotechnology, Berkeley, California, USA, pp. $165-168$

Moghe R, Divan D and Lambert F 2011 Powering low-cost utility sensors using energy harvesting. Proceedings of 14th European Conference on Power Electronics and Applications, pp. 1-10

Moghe R, Yang Y, Lambert F and Divan D 2010 Design of a low cost self powered "Stick-on" current and temperature wireless sensor for utility assets. Proceedings of IEEE Energy Conversion and Congress and Exposition, pp. 4453-4460

Poonam and Mulge Y 2013 Remote temperature monitoring using LM35 sensor and intimate android user via C2DM service. Int. J. Comput. Sci. Mobile Comput. 2: 32-36

Rong J and Zuozuo G 2005 The application of DS18B20 in thermometric system. J. Agric. Mech. Res. 2005(1): 224-226

Sensaphone remote monitoring system 2009 A brochure on a wireless sensor node [online]. Available from http://www.sensaphone.asia/downloads/LIT-1007_WirelessNode.pdf. [Accessed 6 September 2014]

Sordiashie E 2012 Electromagnetic harvesting to power energy management sensors in the built environment. MSc dissertation, University of Nebraska-Lincoln, USA

Veerasingam S, Karodi S, Shukla S and Yeleti M C 2009 Design of wireless sensor network node on zigbee for temperature monitoring. Intl. Conf. on Advances in Computing, Control \& Telecommunication Technologies (ACT '09), pp. 20-23. doi: 10.1109/ACT.2009.14

Wang W, Cionca V, Wang N, Hayes M, O'Flynn B and O'Mathuna C 2013 Thermoelectric energy harvesting for building energy management wireless sensor networks. Int. J. Distrib. Sensor Netw. 2013: 232438. doi: $10.1155 / 2013 / 232438$

Zhao X, Li W, Zhou L, Song G-B, Ba Q and Ou J 2013 Active thermometry based DS18B20 temperature sensor network for offshore pipeline scour monitoring using K-means clustering algorithm. Int. J. Distrib. Sensor Netw. 2013: 852090, 11 pages. doi: 10.1155/2013/852090 